\title{
Maximum Degree Growth of the Iterated Line Graph
}

\author{
Stephen G. Hartke* \\ Aparna W. Higgins ${ }^{\dagger}$ \\ Department of Mathematics \\ University of Dayton \\ Dayton, OH 45469-2316
}

Submitted: February 25, 1999; Accepted: June 24, 1999.

\begin{abstract}
Let $\Delta_{k}$ denote the maximum degree of the $k^{\text {th }}$ iterated line graph $L^{k}(G)$. For any connected graph $G$ that is not a path, the inequality $\Delta_{k+1} \leq 2 \Delta_{k}-2$ holds. Niepel, Knor, and Šoltés [3] have conjectured that there exists an integer $K$ such that, for all $k \geq K$, equality holds; that is, the maximum degree $\Delta_{k}$ attains the greatest possible growth. We prove this conjecture using induced subgraphs of maximum degree vertices and locally maximum vertices.
\end{abstract}

Mathematics Subject Classification: Primary 05C75, Secondary 05C12.

\section{Introduction}

The line graph $L(G)$ of a graph $G$ is defined as the graph whose vertices are the edges of $G$ and where two vertices in $L(G)$ are adjacent if and only if the corresponding edges in $G$ are incident to a common vertex. Line graphs are well studied, and we direct the reader to [1] for a general discussion of the properties of line graphs. In particular, if $v$ is a vertex in $L(G)$ and $u$ and $w$ are the endpoints of the edge in $G$ that corresponds to $v$, then $\operatorname{deg}_{L(G)}(v)=\operatorname{deg}_{G}(u)+\operatorname{deg}_{G}(w)-2$. Thus, the maximum degree $\Delta(L(G))$ of $L(G)$ satisfies

$$
\Delta(L(G)) \leq 2 \Delta(G)-2,
$$

*Correspondence to: 3252 Greenmount Dr., Cincinnati, OH 45248-3940

$\dagger$ 'email: higgins@saber . udayton. edu 
and the minimum degree $\delta(L(G))$ satisfies

$$
\delta(L(G)) \geq 2 \delta(G)-2 .
$$

The iterated line graph $L^{k}(G)$ is defined recursively as $L^{0}(G)=G$, and $L^{k}(G)=$ $L\left(L^{k-1}(G)\right)$ for $k \geq 1$. Though much is known about the line graph, few results are known for the iterated line graph. Some of these results can be found in [2]. Using the inequalities above, Niepel, Knor, and Šltés [3] developed the following bounds for the maximum degree $\Delta_{k}$ and minimum degree $\delta_{k}$ of the iterated line graph $L^{k}(G)$ :

$$
2^{k}\left(\delta_{0}-2\right)+2 \leq \delta_{k} \leq \Delta_{k} \leq 2^{k}\left(\Delta_{0}-2\right)+2 .
$$

Thus, the maximum and minimum degree both have order $\Theta\left(2^{k}\right)$.

Niepel, et al., have conjectured that the maximum degrees of all iterated line graphs, with the exception of paths, eventually attain the maximum growth rate of $\Delta_{k+1}=2 \Delta_{k}-2$. In this paper, we say that a graph $G$ has the Maximum Degree Growth Property (MDGP) if $\Delta(L(G))=2 \Delta(G)-2$. Thus, the conjecture states that there exists an integer $K$ such that, for all $k \geq K, L^{k}(G)$ possesses the MDGP. The focus of this paper is to present a proof of this conjecture.

In the following work, only finite simple connected graphs with no loops are considered. Note that the iterated line graph of a path eventually becomes the empty graph and that the iterated line graphs of cycles and $K_{1,3}$ (whose line graph is a triangle) trivially satisfy the conjecture. Therefore, we consider only graphs that are not contained in these classes.

\section{The Maximum Degree Induced Subgraph}

We begin with some basic definitions.

Definition 1. Let $\Delta(G)$ be the maximum degree among the vertices of $G$, and $\delta(G)$ be the minimum degree. Let $\Delta_{k}$ denote $\Delta\left(L^{k}(G)\right)$ and $\delta_{k}$ denote $\delta\left(L^{k}(G)\right)$.

Definition 2. A graph $G$ has the Maximum Degree Growth Property (MDGP) if $\Delta(L(G))=2 \Delta(G)-2$.

The conjecture of Niepel, Knor, and Šoltés can now be stated as follows.

Conjecture 3. [3] Let $G$ be a connected graph that is not a path. Then there exists an integer $K$ such that, for all $k \geq K, L^{k+1}(G)$ has the MDGP; that is,

$$
\Delta_{k+1}=2 \Delta_{k}-2
$$

We now introduce the maximum degree induced subgraph $M(G)$ which will enable us to characterize those graphs $G$ possessing the MDGP. 
Definition 4. Let the maximum degree induced subgraph $M(G)$ be the subgraph of $G$ induced by the vertices of $G$ that have maximum degree $\Delta(G)$. Let $M_{k}$ denote $M\left(L^{k}(G)\right)$.

Lemma 5. The MDGP holds for a graph $G$ if and only if $M(G)$ contains an edge.

Proof. If $M(G)$ contains an edge then two vertices of degree $\Delta(G)$ are adjacent, and the edge joining these vertices will create a vertex of degree $\Delta(L(G))=2 \Delta(G)-2$ in $L(G)$.

Inversely, if $M(G)$ does not contain an edge, then no two vertices of degree $\Delta(G)$ are adjacent, and $\Delta(L(G))<2 \Delta(G)-2$.

Note that Lemma 5 implies that the MDGP holds for all $L^{k}(G), k \geq 0$, if and only if $M_{k}$ contains at least one edge for all $k \geq 0$.

The following lemma is a well-known result on line graphs, and is stated without proof.

Lemma 6. If $H$ is a subgraph of $G$, then $L(H)$ is an induced subgraph of $L(G)$.

We can now prove another characterization of graphs for which the MDGP holds.

Lemma 7. The $M D G P$ holds for a graph $G$ if and only if $L(M(G)) \cong M(L(G))$.

Proof. Assume that the MDGP holds for $G$. Then $M(G)$ has an edge, and by Lemma $6, L(M(G))$ is a nonempty subgraph of $L(G)$. Since every edge in $M(G)$ is incident to two vertices of degree $\Delta(G)$, then for all vertices $v \in L(M(G))$, $\operatorname{deg}_{L(G)}(v)=$ $2 \Delta(G)-2=\Delta(L(G))$ and thus $v \in M(L(G))$. Suppose $v$ is a vertex in $M(L(G))$. Since $\operatorname{deg}_{L(G)}(v)=\Delta(L(G))=2 \Delta(G)-2$, the edge $e$ corresponding to $v$ in $M(L(G))$ must be incident to two vertices in $G$ of degree $\Delta(G)$. Thus, $e$ is an edge in $M(G)$, and $v \in L(M(G))$. Therefore, the vertex sets of $M(L(G))$ and $L(M(G))$ are equal. Since both $M(L(G))$ and $L(M(G))$ are induced subgraphs of $L(G)$, the adjacencies of $M(L(G))$ and $L(M(G))$ are exactly the same. Therefore, $L(M(G)) \cong M(L(G))$.

Inversely, assume that the MDGP does not hold for $G$. By Lemma $5, M(G)$ does not contain an edge. Thus, $L(M(G))$ is defined on an empty vertex set. But $M(L(G))$ cannot be the empty graph since $L(G)$ is a finite non-empty graph $(G$ is not a path or a single vertex) and at least one vertex in $L(G)$ must have maximum degree. Therefore, $M(L(G)) \varsubsetneqq L(M(G))$.

Corollary 8. If $L^{k}(M(G))$ contains an edge for all $k \geq 0$, then the MDGP will hold for all $L^{k}(G), k \geq 0$.

Proof. If $L^{k}(M(G))$ contains an edge for all $k \geq 0$, then, by Lemma $7, M_{k}$ contains an edge for all $k \geq 0$, and the result follows by Lemma 5 . 
Corollary 8 proves the conjecture for many graphs, since we only need to consider whether or not $L^{k}(M(G))$ always has an edge for $k \geq 0$. Paths and (vertex-disjoint) unions of paths are the only graphs that do not satisfy this condition, and thus the maximum degree growth of graphs whose $M(G)$ are paths or unions of paths remains unresolved with the techniques presented thus far. The concept of the maximum degree induced subgraph is insufficient for these cases because it provides no information about $M_{k+1}$ if $M_{k}$ does not contain an edge. In the next section, we introduce the concept of a local maximum induced subgraph in order to resolve these difficulties.

\section{The Local Maximum Induced Subgraph}

Definition 9. Let the neighborhood $N_{G}(v)$ of a vertex $v$ in $G$ be the set of vertices in $G$ adjacent to $v$. Note that $v \notin N_{G}(v)$.

Let the neighborhood $N_{G}(S)$ of a subgraph $S$ of $G$ be the set of vertices adjacent to vertices in $S$ but not contained in $S$. Thus, $N_{G}(S)=\left(\cup\left\{N_{G}(v): v \in S\right\}\right) \backslash S$.

Definition 10. A vertex $v$ in $G$ is a local maximum if $\operatorname{deg}_{G}(v) \geq \operatorname{deg}_{G}(w)$ for all $w \in N_{G}(v)$.

If $u$ and $w$ are the endpoints of an edge $e$ in $G$, then $u w$ is an alternative notation for the vertex $v \in L(G)$ that corresponds to $e$. This notation suggests the following definition.

Definition 11. A vertex $v \in L(G)$ is generated by a vertex $u \in G$ if the edge $e$ in $G$ that corresponds to $v$ is incident to $u$. A subgraph $J$ of $L(G)$ is generated by a subgraph $H$ of $G$ if, for each vertex $v \in J, v$ is generated by a vertex in $H$.

Note that for a given $J, H$ is in general not unique.

Lemma 12. Every local maximum $v$ in $L(G)$ is generated by a local maximum $w$ in $G$. Moreover, $v$ is generated by $w$ and a vertex in $G$ that is maximum in $N_{G}(w)$.

Proof. Suppose $v$ is in $L(G)$ and $v$ is generated by $x, y \in G$, where $x, y$ are not local maxima in $G$. Assume that $\operatorname{deg}_{G}(x) \leq \operatorname{deg}_{G}(y)$. Then there exists a vertex $w \in N_{G}(y)$ such that $\operatorname{deg}_{G}(w)>\operatorname{deg}_{G}(y)$. The vertex $w y$ in $L(G) \operatorname{has} \operatorname{deg}_{L(G)}(w y)=$ $\operatorname{deg}_{G}(w)+\operatorname{deg}_{G}(y)-2>\operatorname{deg}_{G}(x)+\operatorname{deg}_{G}(y)-2=\operatorname{deg}_{L(G)}(v)$. Vertex $w y$ is in $N_{L(G)}(v)$; therefore, $v$ is not a local maximum.

For the second part of the lemma, assume that $v$ is a local maximum in $L(G)$, where $v$ is generated by $w, y \in G, w$ is a local maximum in $G$ and $y$ is not maximum in $N_{G}(w)$. There exists a vertex $z \in N_{G}(w)$ such that $\operatorname{deg}_{G}(z)>\operatorname{deg}_{G}(y)$. The vertex $w z$ in $L(G)$ has $\operatorname{deg}_{L(G)}(w z)=\operatorname{deg}_{G}(w)+\operatorname{deg}_{G}(z)-2>\operatorname{deg}_{G}(w)+\operatorname{deg}_{G}(y)-2=$ $\operatorname{deg}_{L(G)}(v)$. Vertex $w z$ is in $N_{L(G)}(v)$; therefore, $v$ is not a local maximum, resulting in a contradiction. 
Definition 13. Let the local maximum induced subgraph $\mathfrak{L M}(G)$ be the subgraph of $G$ induced by local maximum vertices. Let $\mathfrak{L M}_{k}$ denote $\mathfrak{L M}\left(L^{k}(G)\right)$.

We now develop some useful properties of the components of $\mathfrak{L} \mathfrak{M}(G)$.

Lemma 14. Let $C$ be a component of $\mathfrak{L M}(G)$. Then all vertices in $C$ have the same degree in $G$.

Proof. Let $u, v$ be adjacent vertices in a component $C$ of $\mathfrak{L M}(G)$, where $\operatorname{deg}_{G}(u) \neq$ $\operatorname{deg}_{G}(v)$. Then $\operatorname{deg}_{G}(u)>\operatorname{deg}_{G}(v)$ and $v$ is not a local maximum, or $\operatorname{deg}_{G}(u)<$ $\operatorname{deg}_{G}(v)$ and $u$ is not a local maximum, generating a contradiction.

Similar to the individual local maxima, the components of $\mathfrak{L M}(L(G))$ can be determined by the components of $\mathfrak{L M}(G)$. This property is shown in the following lemmas.

Lemma 15. If a component $C$ of $\mathfrak{L M}(G)$ contains an edge, then $L(C)$ is a component of $\mathfrak{L} \mathfrak{M}(L(G))$.

Proof. By Lemma 12, every vertex in $\mathfrak{L M}(L(G))$ is generated by a vertex in $\mathfrak{L M}(G)$. Let $C$ be a component of $\mathfrak{L M}(G)$ that contains an edge, where $\operatorname{deg}_{G}(v)=r$ for all $v \in C$. If $v$ and $w$ are adjacent vertices in $C$, then the degree of the vertex $v w$ in $L(G)$ is $\operatorname{deg}_{L(G)}(v w)=2 r-2$. By Lemma 6 , and since the line graph preserves connectivity, $L(C)$ is a connected induced subgraph of $L(G)$, where every vertex in $L(C)$ has degree $2 r-2$.

To complete the proof of the lemma, we show that no vertex in $N_{L(G)}(L(C))$ is a local maximum. Every vertex $v y$ in $N_{L(G)}(L(C))$ is generated by a vertex $y \in N_{G}(C)$ that is adjacent to a vertex $v \in C$. If $\operatorname{deg}_{G}(y)<r$, then $\operatorname{deg}_{L(G)}(v y)<2 r-2$, and $v y$ is not a local maximum in $L(G)$. If $\operatorname{deg}_{G}(y)=r$, then since $y \notin C$ there exists a vertex $z \in N_{G}(y) \backslash C$ such that $\operatorname{deg}_{G}(z)>r$. Then $\operatorname{deg}_{L(G)}(y z)>2 r-2=\operatorname{deg}_{L(G)}(v y)$, and $v y$ is not a local maximum in $L(G)$. Therefore, no vertex in $N_{L(G)}(L(C))$ is a local maximum, and thus $L(C)$ is a component of $\mathfrak{L} \mathfrak{M}(L(G))$.

Lemma 16. Let $C$ be a component of $\mathfrak{L M}(G)$ that does not contain an edge $(C$ consists solely of a vertex $v$ ), and let $s$ be the maximum degree of vertices in $N_{G}(v)$. Then the following hold:

1. If $s=\operatorname{deg}_{G}(v)$, then no vertex in $L(G)$ generated by $v$ is a local maximum.

2. If $s<\operatorname{deg}_{G}(v)$, and if for all $u \in N_{G}(v)$ with $\operatorname{deg}_{G}(u)=s$ there exists $w \in$ $N_{G}(u) \backslash\{v\}$ such that $\operatorname{deg}_{G}(w)>\operatorname{deg}_{G}(v)$, then no vertex in $L(G)$ generated by $v$ is a local maximum.

3. If $s<\operatorname{deg}_{G}(v)$, and if for a given $u \in N_{G}(v)$ with $\operatorname{deg}_{G}(u)=s, \operatorname{deg}_{G}(w)<$ $\operatorname{deg}_{G}(v)$ for all $w \in N_{G}(u) \backslash\{v\}$, then $v$ will generate a local maximum in $L(G)$ for each such u. All local maxima thus generated will be adjacent. 
4. If $s<\operatorname{deg}_{G}(v)$, and if for a given $u \in N_{G}(v)$ with $\operatorname{deg}_{G}(u)=s$ there exists $w \in N_{G}(u) \backslash\{v\}$ such that $\operatorname{deg}_{G}(w)=\operatorname{deg}_{G}(v)$, then $v$ will generate locally maximum vertices in $L(G)$ that are adjacent, and the locally maximum vertices generated by $v$ will be adjacent to other locally maximum vertices in $L(G)$ that are generated by a different component in $\mathfrak{L M}(G)$.

Proof. Let $\operatorname{deg}_{G}(v)=r$, and let $u \in N_{G}(v)$ have maximum degree in $N_{G}(v)$. Note that all local maxima in $L(G)$ generated by $v$, if any, will be adjacent to each other.

Let $y \in N_{G}(v)$, where $\operatorname{deg}_{G}(y)<s$. So $y$ is not maximum in $N_{G}(v)$. Then $\operatorname{deg}_{L(G)}(v y)<r+s-2=\operatorname{deg}_{L(G)}(v u)$, and $v y$ is not a local maximum in $L(G)$. Thus, only vertices generated by $v$ and vertices maximum in $N_{G}(v)$ can be locally maximum in $L(G)$.

Assume that $\operatorname{deg}_{G}(u)=r=\operatorname{deg}_{G}(v)$. Since $u \notin C$ there exists a vertex $w \in$ $N_{G}(u) \backslash\{v\}$ such that $\operatorname{deg}_{G}(w)>r$. Then $\operatorname{deg}_{L(G)}(u w)>2 r-2=\operatorname{deg}_{L(G)}(v u)$, and $v u$ is not a local maximum in $L(G)$, proving part 1 .

Assume that $r=\operatorname{deg}_{G}(v)>\operatorname{deg}_{G}(u)=s$. If there exists a vertex $w \in N_{G}(u) \backslash\{v\}$ such that $\operatorname{deg}_{G}(w)>r$, then $\operatorname{deg}_{L(G)}(u w)>r+s-2=\operatorname{deg}_{L(G)}(v u)$, and $v u$ is not a local maximum in $L(G)$. If such a vertex $w$ exists for all maximum degree vertices in $N_{G}(v)$, then no vertex in $L(G)$ generated by $v$ is a local maximum, proving part 2 . If $\operatorname{deg}_{G}(w) \leq r$ for all $w \in N_{G}(u) \backslash\{v\}$, then $\operatorname{deg}_{L(G)}(v u)=r+s-2 \geq \operatorname{deg}_{L(G)}(u w)$, and $v u$ is a local maximum in $L(G)$. This proves part 3. If there exists a vertex $w \in N_{G}(u) \backslash\{v\}$ such that $\operatorname{deg}_{G}(w)=r$, then $\operatorname{deg}_{L(G)}(u w)=r+s-2=\operatorname{deg}_{L(G)}(v u)$, and $v u$ and $u w$ are adjacent local maxima in $L(G)$, proving part 4 . Note that vertices $v u$ and $u w$ are in the same component of $\mathfrak{L M}(L(G))$, but the component is generated by more than one component in $\mathfrak{L M}(G)$.

Thus, we now know the possible effects of the line graph on components of $\mathfrak{L M}_{k}$. However, we can determine the exact effect on a component of $\mathfrak{L M}_{k}$ if $k$ is large enough.

Corollary 17. Each component of $\mathfrak{L M}(L(G))$ is generated by components of $\mathfrak{L M}(G)$, and each component of $\mathfrak{L M}(G)$ generates at most one component of $\mathfrak{L M}(L(G)$ ).

Proof. The first part of the corollary follows from Lemma 12, and the second part follows from Lemmas 15 and 16.

Corollary 18. There exists an integer $J_{1}$ such that every component of $\mathfrak{L M}_{k}$ generates a component of $\mathfrak{L M}_{k+1}$ for $k \geq J_{1}$, and every component of $\mathfrak{L M}_{k+1}$ is generated by exactly one component of $\mathfrak{L M}_{k}$.

Proof. Let $c_{k}$ denote the number of components of $\mathfrak{L M}_{k}$. By Corollary 17, the sequence $\left\{c_{k}\right\}$ is non-increasing. Since $G$ is a finite graph, there exist vertices of maximum degree, and thus $\mathfrak{L M}_{k}$ is nonempty and $c_{k} \geq 1$ for all $k$. Therefore, there exists an integer $J_{1}$ such that $c_{k}$ is constant for $k \geq J_{1}$. Using Corollary 17, the statement of this corollary follows. 
The following notation will allow us to consider a particular component $C_{J_{1}}$ of $\mathfrak{L M}_{J_{1}}$ and the components in $\mathfrak{L M}_{k}$ that $C_{J_{1}}$ generates for $k>J_{1}$.

Definition 19. Let $J_{1}$ be the integer given in Corollary 18, and let $\left\{C_{k}\right\}_{k \geq J_{1}}$ be a sequence such that $C_{k}$ is a component of $\mathfrak{L M}_{k}$ and $C_{k+1}$ is generated by $C_{k}$. Let $r_{k}=\operatorname{deg}_{L^{k}(G)}(v)$ for all $v \in C_{k}$.

Lemma 20. For $k \geq J_{1}$ given in Corollary 18, let $u \in N_{L^{k}(G)}\left(C_{k}\right)$ where $\operatorname{deg}_{L^{k}(G)}(u)=$ a. Then there exists a vertex $v \in L^{k+1}(G)$ generated by $u$ where $\operatorname{deg}_{L^{k+1}(G)}(v)=$ $r_{k}+a-2$, and either $v \in N_{L^{k+1}(G)}\left(C_{k+1}\right)$ or $v \in C_{k+1}$.

Proof. Let $w$ be a vertex in $C_{k}$ that is adjacent to $u \in N_{L^{k}(G)}\left(C_{k}\right)$. Then the degree of $v=u w$ in $L^{k+1}(G)$ is $r_{k}+a-2$. Since $C_{k}$ generates $C_{k+1}$, there exists a vertex $z \in N_{L^{k}(G)}(w)$ such that $w z \in C_{k+1}$. If $\operatorname{deg}_{L^{k}(G)}(u)=\operatorname{deg}_{L^{k}(G)}(z)$, then $v \in C_{k+1}$; otherwise, $v \in N_{L^{k+1}(G)}\left(C_{k+1}\right)$.

Note that if $C_{k}$ has an edge, then

$$
\begin{aligned}
r_{k+1}-\operatorname{deg}_{L^{k+1}(G)}(v) & =\left(2 r_{k}-2\right)-\left(r_{k}+a-2\right) \\
& =r_{k}-a .
\end{aligned}
$$

If $C_{k}$ does not have an edge, then

$$
\begin{aligned}
r_{k+1}-\operatorname{deg}_{L^{k+1}(G)}(v) & <\left(2 r_{k}-2\right)-\left(r_{k}+a-2\right) \\
& =r_{k}-a .
\end{aligned}
$$

Note also that if two vertices $u_{1}$ and $u_{2}$ in $L^{k}(G)$ are adjacent to a common vertex $w$ in $C_{k}$, then vertices $v_{1}=u_{1} w$ and $v_{2}=u_{2} w$ are adjacent in $L^{k+1}(G)$.

The following lemma is another well-known result on iterated line graphs, and is presented without proof.

Lemma 21. Let $G$ be a connected graph that is not a path, cycle, or $K_{1,3}$. Then for all integers $q$ there exists an integer $Q$ such that $\delta_{Q}>q$.

We now proceed with our characterization of the components of $\mathfrak{L M}_{k}$.

Lemma 22. There exists an integer $J_{2}$ such that $C_{J_{2}}$ contains an edge.

Proof. Assume that there does not exist an integer $J_{2}$ such that $C_{J_{2}}$ contains an edge. Assume that $J_{1}$ given in Corollary 18 is large enough such that, by Lemma $21, \delta_{J_{1}}>1$. Then $r_{J_{1}}>r_{J_{1}}-\delta_{J_{1}}+1$. Since $r_{J_{1}}-\delta_{J_{1}}+1$ represents the number of possible degrees a vertex in $N_{L^{J_{1}}(G)}\left(C_{J_{1}}\right)$ can have, by the Pigeonhole Principle there exist two vertices of the same degree adjacent to every vertex in $C_{J_{1}}$. Let $w \in C_{J_{1}}$. Then there are two vertices $x_{J_{1}}$ and $y_{J_{1}}$ of degree $a_{J_{1}}$ adjacent to $w$. Construct a sequence of pairs of vertices $\left\{\left(x_{k}, y_{k}\right)\right\}$, where $x_{k+1}$ is generated by $x_{k}$ as described in 
Lemma 20 and similarly for $y_{k+1}$. Thus, the degree $a_{k+1}$ of $x_{k+1}$ and $y_{k+1}$ is given by $a_{k+1}=a_{k}+r_{k}-2$. Both vertices in each pair $\left(x_{k}, y_{k}\right)$ are adjacent to one another for $k>J_{1}$, since $x_{k-1}$ and $y_{k-1}$ are adjacent to a common vertex in $C_{k-1}$. Since, for $k \geq J_{1}, C_{k}$ does not contain an edge, $r_{k+1}-a_{k+1}<r_{k}-a_{k}$. Thus, there must exist an integer $P$ where $r_{P}-a_{P}=0$. This implies that $C_{P}$ contains two vertices and thus must contain an edge, which is a contradiction.

Lemma 23. If there does not exist an integer $P$ such that $C_{k}$ contains an edge for all $k \geq P$, then there exists an integer $J_{3}$ such that there are three vertices in $N_{L^{J_{3}(G)}}\left(C_{J_{3}}\right)$ where all three vertices have the same degree and are adjacent to the same vertex in $C_{J_{3}}$.

Proof. Assume that there does not exist an integer $P$ such that $C_{k}$ contains an edge for all $k \geq P$. By Lemma 22, there exists an integer $J_{2}$ such that $C_{J_{2}}$ contains an edge. Let $J_{3}$ be the smallest integer greater than $J_{2}$ such that $C_{J_{3}}$ does not contain an edge. Then $C_{J_{3}-1}$ contains exactly one edge and two vertices. Let $b_{k}$ denote the minimum degree of vertices in $N_{L^{k}(G)}\left(C_{k}\right)$. The component $C_{J_{3}-1}$ generates $C_{J_{3}}$, where $C_{J_{3}}$ consists of the single vertex $w$ of degree $r_{J_{3}}=2 r_{J_{3}-1}-2$. The minimum degree of vertices in $N_{L^{J_{3}(G)}}(w)$ is $b_{J_{3}}=r_{J_{3}-1}+b_{J_{3}-1}-2$. By Lemma 21, the minimum degree $\delta_{k}$ can be made arbitrarily large by iterating long enough, and thus $b_{J_{3}-1} \geq \delta_{J_{3}-1}>2$ can be assumed. Then

$$
\begin{array}{rlrl}
b_{J_{3}-1} & >2 & & \\
\Rightarrow & r_{J_{3}-1}-1 & >r_{J_{3}-1}-b_{J_{3}-1}+1 \\
\Rightarrow & 2 r_{J_{3}-1}-2 & >2\left(\left(2 r_{J_{3}-1}-2\right)-\left(r_{J_{3}-1}+b_{J_{3}-1}-2\right)+1\right) \\
\Rightarrow & & r_{J_{3}} & >2\left(r_{J_{3}}-b_{J_{3}}+1\right) .
\end{array}
$$

Since $r_{J_{3}}-b_{J_{3}}+1$ represents the maximum number of degrees a vertex in $N_{L^{J_{3}(G)}}\left(C_{J_{3}}\right)$ can have, by the Pigeonhole Principle and inequality $(*), w$ has at least three adjacent vertices that have the same degree.

Lemma 24. There exists an integer $J_{4}$ such that $C_{k}$ contains an edge for all $k \geq J_{4}$.

Proof. Assume that there does not exist an integer $P$ such that $C_{k}$ contains an edge for all $k \geq P$. Then by Lemma 23 there exists an integer $J_{3}$ such that there are three vertices $x_{J_{3}}, y_{J_{3}}$, and $z_{J_{3}}$ in $N_{L^{J_{3}(G)}}\left(C_{J_{3}}\right)$ where all three vertices have the same degree $a_{J_{3}}$ and are adjacent to the same vertex $w$ in $C_{J_{3}}$. Construct a sequence of triples of vertices $\left\{\left(x_{k}, y_{k}, z_{k}\right)\right\}$, where $x_{k+1}$ is generated by $x_{k}$ as described in Lemma 20 and similarly for $y_{k+1}$ and $z_{k+1}$. Thus, the degree $a_{k+1}$ of $x_{k+1}, y_{k+1}$, and $z_{k+1}$ is given by $a_{k+1}=a_{k}+r_{k}-2$. The vertices in each triple $\left(x_{k}, y_{k}, z_{k}\right)$ are adjacent to one another for $k>J_{3}$, since $x_{k-1}, y_{k-1}$, and $z_{k-1}$ are adjacent to a common vertex in $C_{k-1}$. Since $C_{k}$ does not contain an edge for all $k \geq J_{3}$, there exists an infinite number of integers $k \geq J_{3}$ where $C_{k}$ does not contain an edge and where $r_{k+1}-a_{k+1}<r_{k}-a_{k}$. Thus, there must exist an integer $Q$ where $r_{Q}-a_{Q}=0$. This implies that $\left(x_{Q}, y_{Q}, z_{Q}\right)$ forms a triangle in $C_{Q}$, and thus $C_{k}$ will contain an edge for all $k \geq Q$. 
THE ELECTRONIC JOURNAL OF COMBINATORICS 6 (1999), \#R28

We now have all the necessary lemmas to prove the conjecture.

Theorem 25. For all connected graphs $G$ that are not paths, there exists an integer $K$ such that the $M D G P$ will hold for $L^{k}(G)$ for all $k \geq K$.

Proof. By Lemma 24 and since $\mathfrak{L M}_{k}$ has a finite number of components, there exists a $K$ such that every component of $\mathfrak{L M}_{k}$ contains an edge for all $k \geq K$. By Lemma $14, M_{k}$ is a subset of the components in $\mathfrak{L M}_{k}$. Thus, $M_{k}$ contains an edge for all $k \geq K$, and, by Lemma 5, the MDGP will hold for all $k \geq K$.

\section{Conclusion}

Theorem 25 proves the existence of an integer $K$ such that the MDGP will hold for all $k \geq K$. From this result, we can conclude that the formula $\Delta_{k}=2^{k-K}\left(\Delta_{K}-2\right)+2$ holds for all $k \geq K$. However, this formula is not particularly useful unless the least integer $K$ such that the MDGP will hold for all $k \geq K$ can be determined. The calculation of this tight bound for a given graph remains an open question.

Niepel, Knor, and Šltés in [3] also posed a corresponding conjecture for the minimum degree $\delta_{k}$ : There exists an integer $P$ such that $\delta_{k+1}=2 \delta_{k}-2$ for $k \geq$ $P$. Even though analogous minimum degree induced subgraphs and local minimum induced subgraphs can be defined, the authors have been unable to prove lemmas corresponding to Lemmas 22 and 23 for the minimum degree case. This conjecture also remains an open question.

\section{Acknowledgments}

The first author wishes to thank the University of Dayton Honors Program for support of his Honors Thesis, of which this work is a part. We also thank the referees for their suggestions.

\section{References}

[1] R. L. Hemminger and L. W. Beineke, "Line Graphs and Line Digraphs," in L. W. Beineke and R. J. Wilson, ed., Selected Topics in Graph Theory, Academic Press, New York, 1978, pp. 271-305.

[2] C. Hoover, "The Behavior of Properties of Graphs under the Line Graph Operation," University of Dayton Honors Thesis, 1991.

[3] L'. Niepel, M. Knor, and L'. Šoltés, "Distances in Iterated Line Graphs," Ars Combinatoria 43 (1996), pp. 193-202. 